Präv Gesundheitsf 2021 · 16:361-367

https://doi.org/10.1007/s11553-020-00818-5

Eingegangen: 19. Juni 2020

Angenommen: 9. November 2020

Online publiziert: 3. Dezember 2020

๑) Der/die Autor(en) 2020

\section{Hintergrund und Fragestellung}

\section{Hintergrund}

Weltweit leben 15-23\% aller Kinder mit einem psychisch erkrankten Elternteil zusammen. Betroffene Kinder unterliegen einer bis $\mathrm{zu} 50 \%$ igen Wahrscheinlichkeit, selbst eine psychische Störung zu entwickeln [8]. Jedes 5. bis 10. Kind psychisch erkrankter Eltern erfüllt die Kriterien für eine Kindeswohlgefährdung [1]. Noch im Erwachsenenleben zeigt sich bei Betroffenen, dass mangelndes Sicherheitsgefühl und Stigmatisierungserfahrungen in der Kindheit sowie familiäre Kommunikationsdefizite sich noch insofern auf ihren Bindungsstil auswirken können, als dass dieser zum ängstlich-vermeidenden Typ tendiert [6]. In den letzten 10 bis 15 Jahren fanden vielfältige Entwicklungen im Bereich der Unterstützung von Familien mit einem psychisch erkrankten Elternteil statt [16]. Die Daten zur Wirksamkeit der bestehenden Interventionen sind heterogen. Es existieren nur wenige evidenzbasierte und empirisch überprüfte Interventionsmaßnahmen [18]. Zudem richten sich Interventionen meist breitgefächert an alle Kinder psychisch erkrankter Eltern bzw. deren Familien. Teilweise werden zwar Interventionen für bestimmte elterliche Störungen angeboten, aber darüber hinausgehend findet keine Differenzierung der Zielgruppe statt [2, 15, 17]. Diesem wenig differenzierten Ansatz scheint die Annahme zugrunde zu liegen, dass Kinder psychisch erkrankter Eltern eine homogene Gruppe darstellen.

Lydia Pfeifer (D)

FH Bielefeld, Bielefeld, Deutschland

\title{
Kinder psychisch erkrankter Eltern als heterogene Zielgruppe
}

\section{Zielsetzung und Fragestellung}

In dieser Studie soll indes ein differenzieller Forschungsansatz verfolgt werden, der eine detaillierte Perspektive auf die Gemeinsamkeiten und Verschiedenheiten von Kindern psychisch erkrankter Eltern vermittelt. Da es sich bei der zu untersuchenden Population um eine Risikogruppe für die Entwicklung psychischer Störungen handelt [10], werden im Sinne der Salutogenese Risiko- und Schutzfaktoren für die Entstehung dieser Störungen erhoben [13]. Eine genaue Beschreibung und Differenzierung einzelner Fälle soll die Gestaltung passgenauer Präventionsmaßnahmen für die Zielgruppe ermöglichen. Die zugrunde liegende Fragestellung dieser Studie lautet: Wielässt sich die Lebenssituation von Kindern psychisch erkrankter Eltern möglichst umfassend beschreiben, sodass Präventionsmaßnahmen passgenau gestaltet werden können?

\section{Studiendesign und Unter- suchungsmethoden}

Zur Beantwortung der Forschungsfrage wurde eine vergleichende Fallstudie durchgeführt. Diese Methode wurde gewählt, da der differenzielle Forschungsansatz im beforschten Feld ein exploratives Vorgehen darstellt. Zudem ermöglicht eine Fallstudie die detaillierte Darstellung der Fälle in ihrer tatsächlichen Plastizität [19]. Die Fälle wurden auf Basis theoretischer Überlegungen aus der Gruppe der Teilnehmenden einer Intervention für Kinder psychisch erkrankter Eltern ausgewählt. Ziel war die Darstellung von Fällen, in denen bestimmte Merkmale besonders typisch ausgeprägt sind. Die Datenbasis für die vergleichende Fallstudie stammt aus drei Quellen:

1. Fragebogen zu Ressourcen im Kindes- und Jugendalter 8-16 [11],

2. Leitfadeninterviews mit den Kindern mit Unterstützung von Karten [20],

3. Dokumentenanalyse von Anmeldebögen zu der Intervention.

Die Fragebogenerhebung diente der Erfassung der personalen und sozialen Ressourcen der Kinder. Im Verlauf eines Jahres wurde der Fragebogen 4-mal ausgefüllt. Die Ressourcen wurden anhand des Mittelwerts der 4 Erhebungen bestimmt, um die Validität der Variable Ressourcenausprägung zu erhöhen. Im Leitfadeninterview wurden die Kinder nach angenehmen und belastenden Lebenserfahrungen, zur Erkrankung ihrer Eltern sowie zu ihren Perspektiven auf das Erwachsen sein gefragt. Die Interviews wurden mithilfe einer zusammenfassenden Inhaltsanalyse ausgewertet [12]. Mithilfe der Anmeldebögen konnten Angaben der Eltern zu sich selbst und zu ihrem Kind erhoben werden.

\section{Ergebnisse und Diskussion}

$$
\text { Fall „Lena“ }
$$

\section{Darstellung der Ergebnisse "Lena" (• Abb. 1)}

Aus dem Interview mit Lena werden drei Zitate vorgestellt, in denen sie über ihre Mutter spricht:

Lena: „Sie [die Mutter] kann schon was mit mir machen. Nur manchmal ist das auch so ein bisschen schwer. Weil sie hatte ja Depressionen oder irgendwie sowas. Ich weiß nicht mehr ganz. Zwei Jahre lang. Und da konnte sie halt nicht im- 


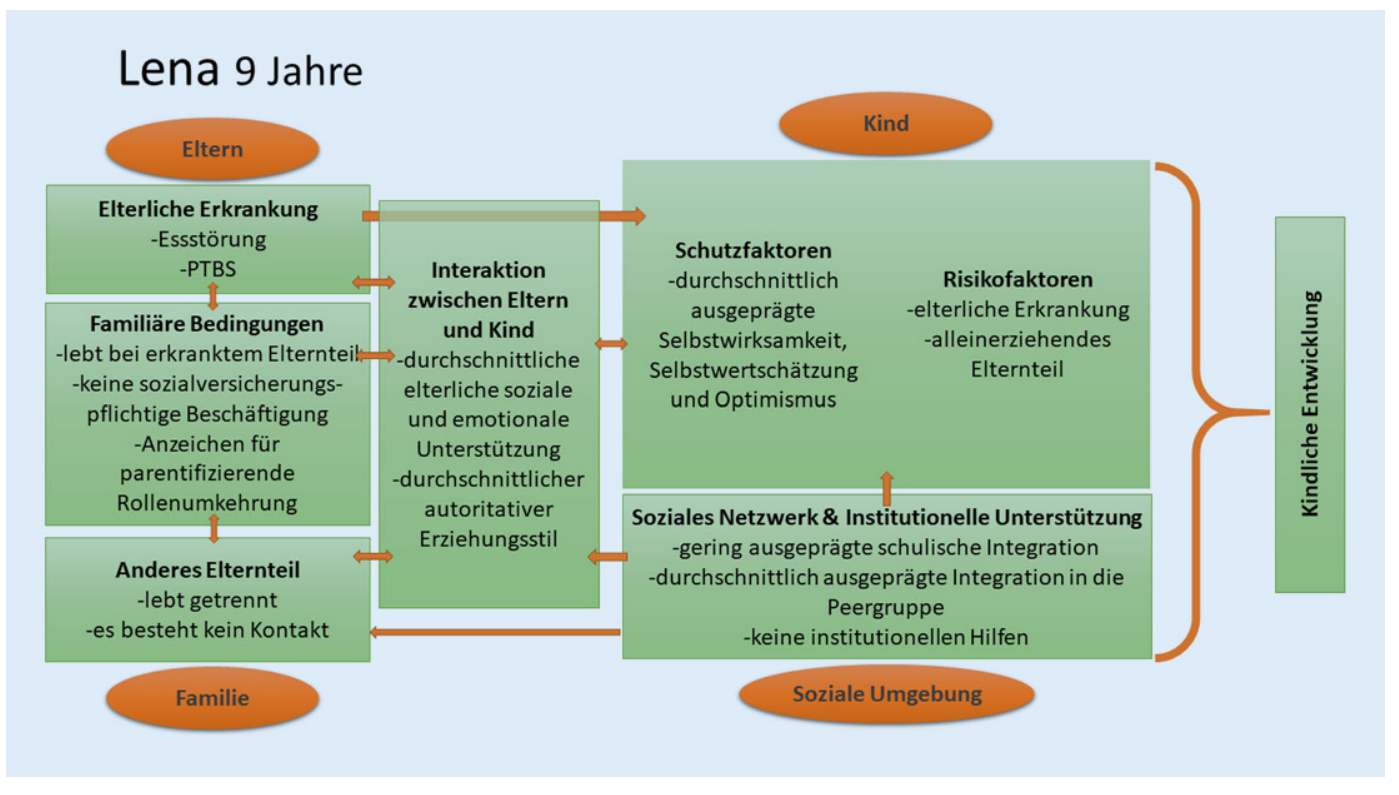

Abb. $1 \varangle$ Darstellung der Merkmalskonstellationen im Hinblick auf Umgebungsbedingungen sowie personale und soziale Risiko- und Schutzfaktoren bei Lena. PTBS Posttraumatische Belastungsstörung. (Abbildung in Anlehnung an Hosman et al. [5]) mer viel machen mit mir. Und das war dann schon manchmal ein bisschen blöd." (Interview 3, Z. 150-157)

Lena: „Wir waren in so einer Klinik ganz lange, sechs Wochen oder so. [...] Und das Blöde war dann, ich konnte im Kindergarten nicht mitmachen, ich hab ganz viel verpasst und ich konnte nicht mit meinen Sachen spielen. Ich konnte da nicht viel essen, weil lecker Essen gab's da eigentlich nicht. [...] Und das war dann [...] blöd, wenn ich da alleine war, weil meine Mutter war dann immer weg und ich war in so einem Kindergarten [...]. Und das war halt immer blöd, wenn man niemanden hat, der so in derselben Größe ist oder mit dem man sich gut versteht." (Interview 3, Z. 159-171)

Lena: „[...] Nur in der ersten Klasse war dann immer unser Problem. Sie [die Mutter] konnte halt nicht so gut aufstehen. Also, das heißt, dass ich halt immer $z u$ spät gekommen bin. Das war halt auch immer so eine Sache. Da hab ich Ärger bekommen von der Lehrerin. Dann hat meine Lehrerin [...] das abgesprochen und dann hat sie mir halt beigebracht, dass ich mir einen Wecker einstellen darf, SOLLTE. [...] Und dann ging's halt gar nicht. In der zweiten Klasse ging es dann. Und in der dritten und der vierten bin ich halt immer rechtzeitig gekommen. War sogar noch zu früh da und ja. Dann ging's wieder." (Interview 3, Z. 173-182)

\section{Diskussion der Ergebnisse "Lena"}

Lena ist von kumulierenden Risikofaktoren betroffen. Dies stellt nach Laucht et al. [7] eine stärkere Entwicklungsgefährdung dar als das Vorliegen einzelner Risikofaktoren. So lebt sie alleine mit ihrem psychisch erkrankten Elternteil zusammen und es besteht kein Kontakt zum anderen Elternteil. Daraus folgt erstens, dass kein psychisch gesundes Elternteil für Lena zur Verfügung steht, was die Abwesenheit eines potenziellen Schutzfaktors darstellt. Zweitens erlebt die Mutter nicht nur die Belastung durch ihre psychische Erkrankung, sondern trägt auch die alleinige elterliche Verantwortung für Lena. Dies kann zu einer hohen Belastung der Mutter führen, was sich wiederum als Risikofaktor auf Lena auswirken kann. Im Interview berichtet Lena von den Auswirkungen der hohen mütterlichen Belastung. So hatte sie sich mehr gemeinsame Aktivitäten mit ihrer Mutter gewünscht. Zudem musste sie bereits in der ersten Klasse selbstständig aufstehen, da sie sonst zu spät zur Schule gekommen wäre. Bei einem Klinikaufenthalt hat Lena die Mutter begleitet, was sie wiederholt als unangenehme Erfahrung bezeichnet. Die Zitate belegen, wie existenziell die Auswirkungen im alltäglichen Leben des Kindes sein können, wenn niemand im Haushalt wohnt, der die versäumten Aufgaben des psychisch erkrankten Elternteils übernimmt. Lena zeigt laut Angaben ihrer
Mutter Anzeichen für eine parentifizierende Rollenumkehr, was in der Fachliteratur als potenzielle Belastung, aber auch als Chance diskutiert wird [3]. Beim Blick auf die Ressourcenausprägung wird deutlich, dass Lena Unterstützung bei der Ausprägung ihrer Empathie und Perspektivenübernahme braucht. Die von Lena als niedrig wahrgenommene schulische Integration deutet auf schulische Probleme hin. Die bei Lena festzustellenden Risikofaktoren „psychische Erkrankung der Eltern“, „alleinerziehendes Elternteil“ und „hohe Belastung der Eltern“ stellen Prädiktoren für das Auftreten von psychischen Problemen bei Kindern dar [22]. Es lässt sich schlussfolgern, dass Kinder, die allein mit ihrem psychisch erkrankten Elternteil zusammenleben einer besonderen Belastung unterliegen und daher besonderer Präventionsmaßnahmen bedürfen.

\section{Fall „Maureen"}

\section{Darstellung der Ergebnisse "Maureen“" (• Abb. 2)}

Mit Maureen konnte leider kein Interview geführt werden, da die Mutter der Interviewteilnahme nicht zugestimmt hat.

\section{Diskussion der Ergebnisse "Maureen"}

Maureen weiß nichts von der Erkrankung ihrer Mutter, da diese die eigene 
Erkrankung vor der Tochter geheim halten möchte. Maureen fühlt sich unterdurchschnittlich in ihre Peergruppe und die schulische Umgebung integriert. Das könnte darauf zurückgeführt werden, dass sie ihre Familie als so konträr zu denen anderer Kinder wahrnimmt, dass sie sich nicht zugehörig fühlt. $\mathrm{Da}$ sie nicht weiß, dass das Verhalten ihrer Mutter einer Krankheit geschuldet ist, kann angenommen werden, dass es ihr schwer fällt, sich ungewöhnliches Verhalten der Mutter zu erklären und einen angemessenen Umgang damit zu finden [9]. Auch ihre Selbstwertschätzung und Selbstwirksamkeit stellen sich unterdurchschnittlich dar, was damit in Zusammenhang gebracht werden kann, dass die Mutter sich aufgrund ihrer Angststörung unberechenbar und inkonsistent verhält. Somit bietet sie für Maureen kein konsequentes und berechenbares Gegenüber. Maureen erlebt sich als wenig wirksam im Kontakt mit der Mutter, da diese sich in einer für ihre Tochter unerklärlichen und nicht zuverlässig beeinflussbaren Weise verhält. Im Kontrast hierzu steht Maureens Einschätzung einer durchschnittlichen elterlichen sozialen und emotionalen Unterstützung sowie eines überdurchschnittlichen autoritativen Erziehungsstils. Dieser scheinbare Gegensatz muss vor dem Hintergrund empirischer Erkenntnisse hinsichtlich der familiären Außendarstellung durch Kinder psychisch erkrankter Eltern diskutiert werden. So neigen die Kinder dazu, die Familie nach außen hin als möglichst normal darzustellen, um diese zu schützen [21]. Das könnte auch bei Maureen der Fall sein und ihre Angaben im Fragebogen in Bezug auf die Mutter beeinflussen. In Maureens Fall zeigt sich ein in der Literatur als typisch für Familien mit einem psychisch erkrankten Elternteil beschriebenes Phänomen: Die Tabuisierung der elterlichen Erkrankung. Wie in diesem Abschnitt hergeleitet wurde, können daraus Folgeprobleme erwachsen, die sich nachteilig auf die gesunde psychische Entwicklung des betroffenen Kindes auswirken können. Psychoedukation kann ein Inhalt von präventiven Interventionen für Kinder psychisch kranker Eltern sein

Präv Gesundheitsf 2021 · 16:361-367 https://doi.org/10.1007/s11553-020-00818-5

(c) Der/die Autor(en) 2020

\section{Kinder psychisch erkrankter Eltern als heterogene Zielgruppe}

\section{Zusammenfassung}

Hintergrund. Kinder, die mit einem psychisch erkrankten Elternteil aufwachsen, unterliegen einer erhöhten Wahrscheinlichkeit selbst psychisch zu erkranken. Der Gestaltung präventiver Interventionen für diese Zielgruppe liegt meist keine differenzielle Perspektive zugrunde.

Ziel der Studie. Ziel der Studie ist die differenzierte Darstellung einzelner Fälle aus der Gruppe „Kinder psychisch erkrankter Eltern". Dies soll einen Beitrag zur Gestaltung passgenauer Interventionsmaßnahmen leisten.

Material und Methoden. Vier Kinder wurden mit dem Fragebogen zu Ressourcen im Kindes- und Jugendalter sowie per Leitfadeninterview befragt. Zudem wurden die Anmeldebögen zu einer Intervention ausgewertet. Diese Daten wurden im Rahmen einer vergleichenden Fallstudie zueinander in Bezug gesetzt.

Ergebnisse und Diskussion. Das Zusammenleben mit einem alleinerziehenden psychisch erkrankten Elternteil kann für Kinder besonders belastend sein. Praktische Unterstützung im Alltag der Familie oder der Beziehungsaufbau zu einer psychisch gesunden Bezugsperson könnte die Kinder entlasten. Die Tabuisierung der elterlichen Erkrankung kann sich nachteilig auf die gesunde psychische Entwicklung von Kindern auswirken, was in psychoedukativen Interventionen für die ganze Familie verändert werden kann, indem offen über die Erkrankung gesprochen wird. Kompensiert das familiäre System die elterliche Erkrankung, sind gegebenenfalls auch die Kinder wenig beeinträchtigt und möglicherweise benötigen sie keine Unterstützung. Kumulieren viele Risikofaktoren, sollte eine Intervention für diese hoch belasteten Kinder auch den Abbau von Risikofaktoren anstreben. Folglich ist beim Zuweisen zu präventiven Interventionen auf die Heterogenität der Zielgruppe im Hinblick auf Risikofaktoren und Ressourcen zu achten.

\section{Schlüsselwörter}

Kinder psychisch kranker Eltern · Vergleichende Fallstudie - Risiko- und Schutzfaktoren . Psychisch kranke Alleinerziehende . Präventive Interventionen gestalten

\section{Children of mentally ill parents as a heterogeneous target group}

\section{Abstract}

Background. Children who grow up with a mentally ill parent are more likely to become mentally ill themselves. The design of preventive interventions for this target group is usually not based on a differential perspective.

Objectives. The aim of the study is to differentiate between individual cases from the group "children of mentally ill parents". This is intended to contribute to the design of tailored interventions.

Materials and methods. Four children were interviewed with the "Fragebogen zu Ressourcen im Kindes- und Jugendalter" as well as in a guided interview. In addition, the registration forms for an intervention were evaluated. These data were triangulated in a comparative case study.

Results and conclusion. Living with a single parent with a mental illness can be particularly stressful for children. Practical support in everyday family life or building a relationship with a mentally healthy adult could provide relieve for the children. Tabooing the parental illness can have a negative impact on the healthy psychological development of children. This can be changed in psychoeducative interventions for the whole family by talking openly about the illness. If the family system compensates for the parental illness, the children may also be less affected and may not need support. If many risk factors accumulate, an intervention for these highly burdened children should also aim to reduce risk factors. Therefore, when allocating to preventive interventions, it is important to take into consideration the heterogeneity of the target group with regard to risk factors and resources.

\section{Keywords}

Children of parents with a mental illness .

Comparative case study $\cdot$ Risk and protective factors · Mentally ill single parents · Designing preventive interventions 


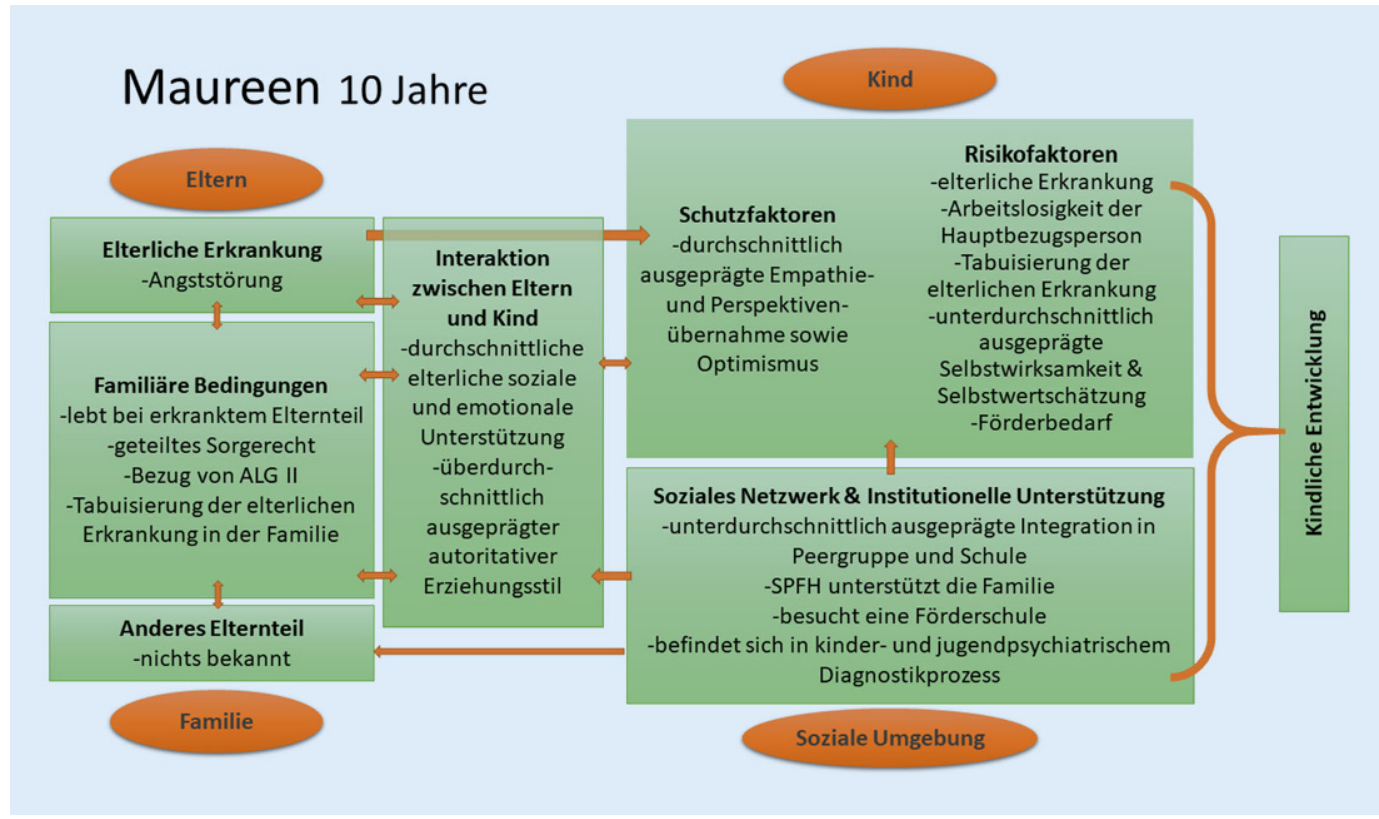

Abb. $2<$ Darstellung der Merkmalskonstellationen im Hinblick auf Umgebungsbedingungen sowie personale und soziale Risiko- und Schutzfaktoren bei Maureen. ALG II Arbeitslosengeld II, SPFH Sozialpädagogische Familienhilfe. (Abbildung in Anlehnung an Hosman et al. [5])

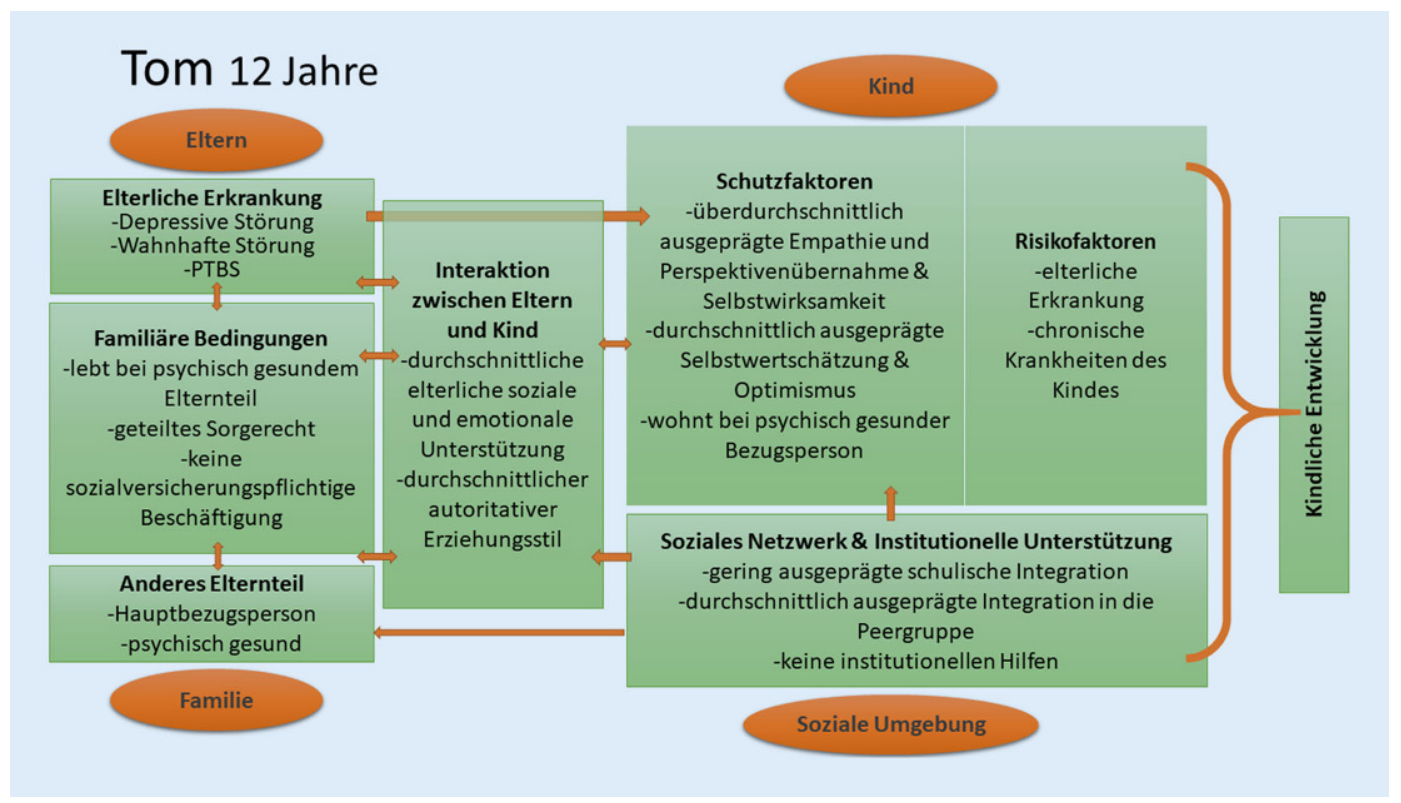

Abb. $3<$ Darstellung der Merkmalskonstellationen im Hinblick auf Umgebungsbedingungen sowie personale und soziale Risiko- und Schutzfaktoren bei Tom. PTBS Posttraumatische Belastungsstörung. (Abbildung in Anlehnung an Hosman et al. [5])

[14]. Insbesondere für Kinder, in deren Familien die elterliche Erkrankung tabuisiert wird, ist Psychoedukation und im Speziellen die Aufklärung über die Erkrankung des Familienmitglieds eine wichtige präventive Maßnahme.

\section{Fall „Tom"}

\section{Darstellung der Ergebnisse „Tom" (० Abb. 3)}

Aus dem Interview mit Tom werden zwei Zitate aus der Kategorie „Belastende Lebenserfahrungen“" vorgestellt.
[...] da wurde ich einmal richtig hart wegen meiner Krankheit gemobbt. Da wurde sich über einen Anfall, den ich bekam wegen einer Unterzuckerung, da zuckte meine Hand ganz dolle, darüber haben sie sich lustig dann gemacht. (Interview 1, Z. 146-152)

Folgendes Zitat bezieht sich auf die Wahrnehmung depressiver Symptome bei sich selbst:

Also was ich blöd fand war, dass es mir im Januar und Februar nicht wirklich gut ging, mir ging es da richtig beschissen/die ganze Zeit einfach nur traurig gewesen und das war das Einzige was wirklich war dann. [...] Ich habe so gut wie immer meine Phasen. Aber manchmal hab ich halt Depression aber manchmal auch nicht. Momentan hab ich keine, aber da fing es mit meinen Depressionen auch richtig an. Das war wirklich nicht schön in der Zeit. Aber mein Vater hat mich unterstützt und meine Schwester. Aber meine Mutter die versteht das nicht so ganz. Also ich hab versucht ihr das zu erklären, ging aber nicht. Mein Vater hat 


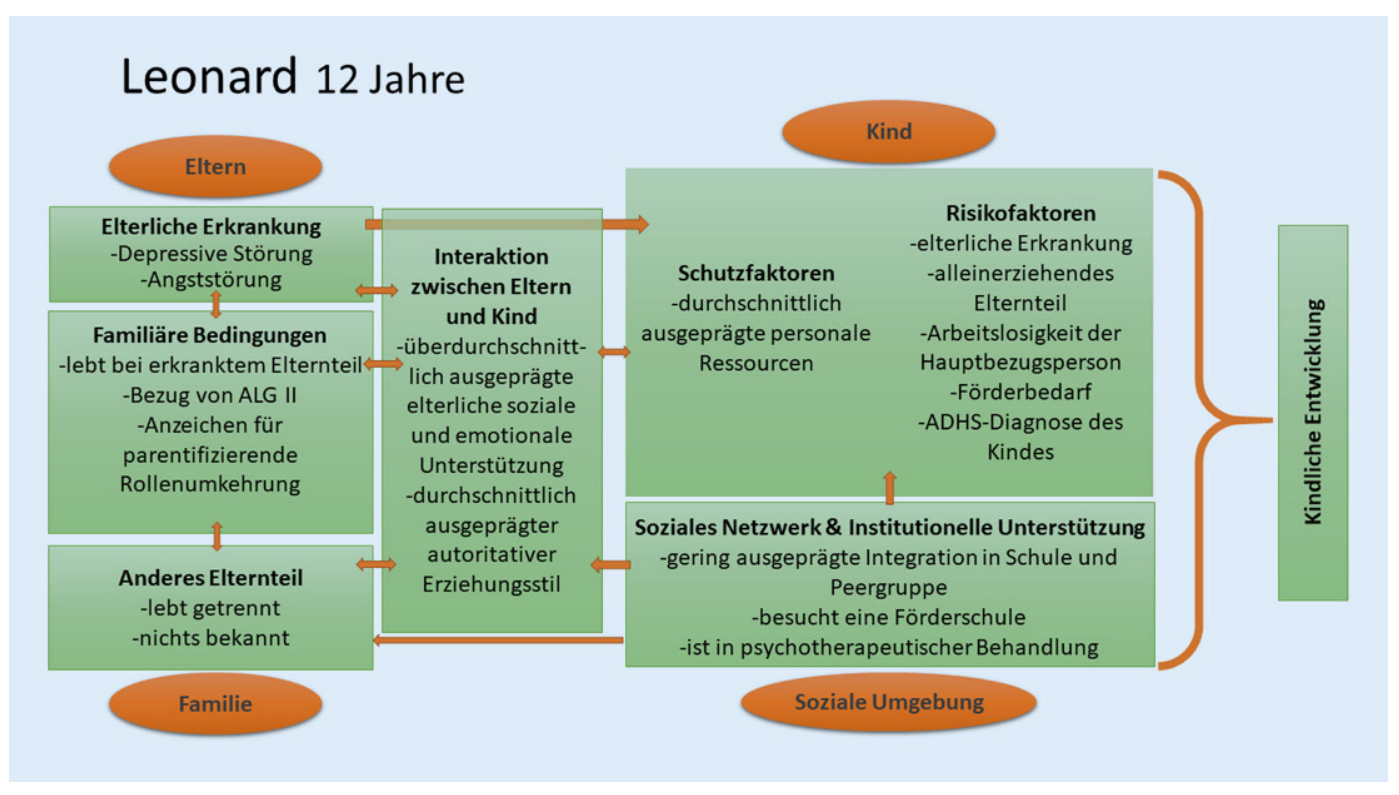

\begin{abstract}
Abb. $4<$ Darstellung der Merkmalskonstellationen im Hinblick auf Umgebungsbedingungen sowie personale und soziale Risiko- und Schutzfaktoren bei Leonard. ALG II Arbeitslosengeld II, ADHS Aufmerksamkeitsdefizit- und Hyperaktivitätsstörung. (Abbildung in Anlehnung an Hosman et al. [5])
\end{abstract}

sie selber. Ja meine Schwester hatte sie mal und konnte mich dabei halt unterstützen. (Interview 1, Z. 293-208)

\section{Diskussion der Ergebnisse „Tom"}

Tom lebt nicht mit seinem psychisch erkrankten Elternteil zusammen. Daher liegt nahe, dass er durch die elterliche Erkrankung weniger stark beeinflusst wird als die anderen Kinder. Dies spiegelt sich auch in der Ausprägung seiner Ressourcen wider, die sich bis auf die schulische Integration als durchschnittlich bis überdurchschnittlich darstellen. Im Interview berichtet er, dass er an sich selbst depressive Symptome wahrgenommen hat, woran sich zeigt, dass er über ein Konzept von Depression als psychischer Erkrankung verfügt. Ganz im Gegensatz zu Maureens Familie scheint in Toms Familie die elterliche Erkrankung ein Gesprächsthema zu sein. Tom beschreibt seine depressive Phase zwar als unangenehm, doch erlebt er den ebenfalls erkrankten Vater und die Schwester als verständnisvolle Unterstützung in Bezug auf seine depressiven Symptome. Im Gegensatz zu den anderen Fällen scheint ein erhöhter Auseinandersetzungsgrad mit psychischen Erkrankungen zu bestehen. Betrachtet man die niedrig ausgeprägte schulische Integration und seinen Bericht über die Ausgrenzungserfahrungen in der Schule, die er im Zusammenhang mit seiner physischen Erkrankung erlebt, scheint hier aus Toms Sicht das dringendere Problem zu liegen. Es lässt sich schlussfolgern, dass die elterliche psychische Erkrankung gegebenenfalls in der Familie bereits gut aufgearbeitet wird, sodass betroffene Kinder keiner Präventionsmaßnahmen in Bezug auf die elterliche Erkrankung bedürfen.

\section{Fall „Leonard"}

\section{Darstellung der Ergebnisse "Leonard" (॰ Abb. 4)}

Die Mutter macht folgende Angaben zum Zusammenleben mit Leonard: Er übernehme viele Aufgaben im Haushalt, so kommeer immer mit zum Einkaufen. Zudem berichtet sie von körperlichen Auseinandersetzungen mit ihrem Sohn. Aus dem Interview mit Leonard wurde folgendes Zitat der Kategorie „Vorstellungen vom Erwachsen sein" zugeordnet.

B: Ähm. Ich soll jetzt mal erzählen, wie ich mir das vorstelle, wenn ich so alt bin wie mein Vater?

\section{I.: Oder deine Mutter, genau.}

[...]

B: Das wär schon stressig.

I: Warum?

B: Weil sie monatlich keine Ahnung Miete bezahlen muss und so, Strom, Wasser.

I: Das ist anstrengend, ne?

B: Ja. Oder Termine.

I: Und denkst du, du würdest das gut hinkriegen oder hättest du dann viel Stress?

\section{B: Sehr viel Stress.}

I: Willst du denn dann erwachsen sein oder eigentlich nicht so unbedingt?

B: Schon, denke ich.

I: Warum?

B: Da muss ich nicht mehr bei meiner Mutter leben. (Interview 2, Z. 15-30)

\section{Diskussion der Ergebnisse "Leonard"}

Leonard lebt mit seiner alleinerziehenden psychisch erkrankten Mutter zusammen, die arbeitslos ist. Das Leben der Mutter nimmt er als stressreich wahr, wie aus dem ausgewählten Interviewzitat hervorgeht, was auf eine Überforderung der Mutter hindeuten kann. Auch der Bericht der Mutter über körperliche Auseinandersetzungen mit ihrem Sohn spricht für elterliche Überforderung. Im Gegensatz dazu steht Leonards durchschnittliche bis überdurchschnittliche Einschätzung des autoritativen Erziehungsstils sowie der elterlichen sozialen und emotionalen Unterstützung. Wie auch bei Lena könnten Leonards Angaben durch seinen Versuch, die Familie zu schützen, beeinflusst werden [21]. Leonards gering ausgeprägte Integration in Schule und Peergruppe deutet daraufhin, dass er in diesen Bereichen keine Ressourcen findet, um seine belastende familiäre Situation auszugleichen. Leonard hat eine ADHSDiagnose sowie Förderbedarf im Bereich emotionale und soziale Entwicklung, was 
eine ungünstige Auswirkung der Risikofaktoren auf seine gesunde Entwicklung nahelegt. Empirische Ergebnisse deuten darauf hin, dass Kinder mit mittlerer Belastung durch Risikofaktoren am meisten von einer Stärkung ihrer Ressourcen profitieren. Wenn die Kinder mehr als drei relevanten Risikofaktoren ausgesetzt sind, kann ein negativer Einfluss auf die psychische Gesundheit nicht mehr durch die Verfügbarkeit von Ressourcen abgepuffert werden. Bei ihnen sollte neben einer Stärkung von Ressourcen auch ein Abbau von Risikofaktoren in Betracht gezogen werden, um ihre gesunde Entwicklung fördern zu können [22]. Bei Leonard kumulieren fünf Risikofaktoren, die seine gesunde psychische Entwicklung beeinträchtigen könnten. Daher haben Präventionsmaßnahmen, die sich an Kinder wie Leonard richten, eher tertiärpräventiven Charakter und sollten auch den Abbau von Risikofaktoren forcieren.

\section{Schlussfolgerungen und Ausblick}

Es konnte anhand von vier unterschiedlichen Fällen die Heterogenität der Zielgruppe im Hinblick auf unterschiedliche Risiko- und Ressourcenprofile aufgezeigt werden. Die Klassifikation als „Kinder psychisch kranker Eltern" reicht somit nicht aus, um den individuellen Unterstützungsbedarf zu erfassen. Der jeweilige Bedarf von Subgruppen konnte aus den Ergebnissen der Fallstudie abgeleitet werden. Diese Erkenntnisse können genutzt werden, um präventive Interventionen für betroffene Kinder passgenau zu gestalten.

Da die untersuchten Fälle im Rahmen einer Intervention für die Zielgruppe ausgewählt wurden, kann die Subgruppe, die durch herkömmliche Interventionen nicht erreicht wird, nicht vorgestellt werden. Vermutlich gehören zu dieser Gruppe sehr stark belastete Kinder, bei denen die Eltern über kein Problembewusstsein verfügen oder sich keine Hilfe holen können. Internetbasierte Interventionen bieten eine Möglichkeit, die Kinder direkt anzusprechen [4]. Im Vergleich zu herkömmlichen Interventionen ist die Gatekeeper-Funktion der Eltern bei internetbasierten Interventionen abgeschwächt oder aufgehoben. Inhalt dieser Interventionen kann die Vermittlung psychoedukativen Wissens, das Anbieten emotionaler Unterstützung und themenbezogenen Austauschs sowie die Vermittlung an Hilfesysteme sein. Weiterführende Forschung ist hier notwendig, um einen bislang unerreichten Teil der Zielgruppe zu erschließen. Die Ergebnisse dieser Studie haben keinen repräsentativen Charakter und sollen als Anregung zu einem differenzierteren Blick auf die Zielgruppe dienen. Somit sollten im Rahmen zukünftiger Arbeiten weitere Subgruppen und ihr jeweiliger Unterstützungsbedarf identifiziert werden.

\section{Fazit für die Praxis}

Kinder psychisch erkrankter Eltern sind eine heterogene Zielgruppe, zu der folgende Subgruppen gehören:

\section{- Psychisch erkrankte Alleinerziehende} Kinder, die allein mit ihrem erkrankten Elternteil zusammenleben, unterliegen einer starken Belastung und können von Interventionen profitieren, die Unterstützung im Alltag bieten oder den Beziehungsaufbau zu einer psychisch gesunden Person ermöglichen.

- Eltern, die ihre Krankheit verheimlichen

Psychoedukative Interventionen können dazu beitragen, den Umgang mit der Erkrankung in der Familie zu verändern und so ungünstige Auswirkungen einer Tabuisierung zu vermeiden.

- Kinder mit Entwicklungsdefiziten Unterliegen Kinder einer erhöhten Belastung und zeigen selbst Entwicklungsdefizite oder psychische Auffälligkeiten, so ist der Abbau von Risikofaktoren anzustreben.

- Wenig beeinträchtigte Kinder

Kann das familiäre System die elterliche Erkrankung kompensieren, so benötigen Kinder gegebenenfalls keine weitere Unterstützung im Hinblick auf die elterliche Erkrankung.

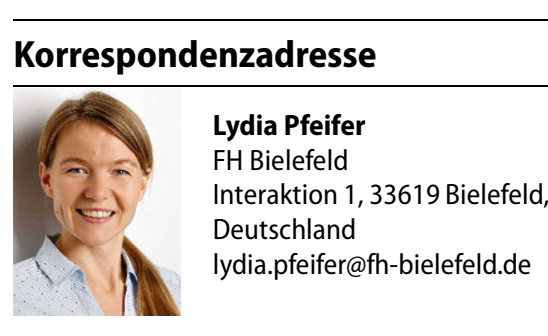

Funding. Open Access funding enabled and organized by Projekt DEAL.

\section{Einhaltung ethischer Richtlinien}

Interessenkonflikt. L. Pfeifer gibt an, dass kein Interessenkonflikt besteht.

Von den Eltern der Interviewpartnerinnen und -partner liegt eine Einverständniserklärung zur Datenerhebung vor. Auch die untersuchten Kinder selbst stimmten der Verwendung ihrer Daten aus der Fragebogenerhebung und des Interviewmaterials zu. Die Datenschutzbestimmungen wurden durchweg eingehalten. Ethische Standards orientierten sich an den Vorgaben der zuständigen Ethikkommission.

Open Access. Dieser Artikel wird unter der Creative Commons Namensnennung 4.0 International Lizenz veröffentlicht, welche die Nutzung, Vervielfältigung, Bearbeitung, Verbreitung und Wiedergabe in jeglichem Medium und Format erlaubt, sofern Sie den/die ursprünglichen Autor(en) und die Quelle ordnungsgemäß nennen, einen Link zur Creative Commons Lizenz beifügen und angeben, ob Änderungen vorgenommen wurden.

Die in diesem Artikel enthaltenen Bilder und sonstiges Drittmaterial unterliegen ebenfalls der genannten Creative Commons Lizenz, sofern sich aus der Abbildungslegende nichts anderes ergibt. Sofern das betreffende Material nicht unter der genannten Creative Commons Lizenz steht und die betreffende Handlung nicht nach gesetzlichen Vorschriften erlaubt ist, ist für die oben aufgeführten Weiterverwendungen des $\mathrm{Ma}$ terials die Einwilligung des jeweiligen Rechteinhabers einzuholen.

Weitere Details zur Lizenz entnehmen Sie bitte der Lizenzinformation auf http://creativecommons.org/ licenses/by/4.0/deed.de.

\section{Literatur}

1. Albermann K, Wiegand-Grefe S, Winter S (2019) Kinderschutz in Familien mit einem psychisch erkrankten Elternteil. Prax Kinderpsychol Kinderpsychiatr 68:6-26

2. Beardslee WR, Wright EJ, Gladstone TRG, Forbes $P$ (2007) Long-term effects from a randomized trial of two public health preventive interventions for parental depression. J Fam Psychol 21:703-713

3. Burton S, Hooper LM, Tomek S, Cauley B, Washington A, Pössel P (2018) The mediating effects of parentification on the relation between parenting behavior and well-being and depressive symptoms. J Child Fam Stud 27:4044-4059 
4. Drost LM, Cuijpers P, Schippers GM (2011) Developing an interactive website for adolescents with a mentally ill family member. Clin Child Psychol Psychiatry 16:351-364

5. Hosman CMH, van Doesum KTM, van Santvoort F (2009) Prevention of emotional problems and psychiatric risks in children of parents with a mental illness in the Netherlands: I. The scientific basis to a comprehensive approach. Aust $\mathrm{EJ} \mathrm{Adv}$ Mental Health 8:250-263

6. Jungbauer J, Kaufmann K, Metz D, Großheinrich N (2019) Bindungsstile bei erwachsenen Kindern psychisch erkrankter Eltern. Psychiatr Prax 46:381-387

7. Laucht M, Esser G, Schmidt MH (2000) Längsschnittforschung zur Entwicklungsepidemiologie psychischer Störungen: Zielsetzung, Konzeption und zentrale Befunde der Mannheimer Risikokinderstudie. ZKlin Psychol Psychother 29:246-262

8. LeijdesdorffS, van Doesum K, Popma A, Klaassen R, van Amelsvoort T (2017) Prevalence of psychopathology in children of parents with mental illness and/or addiction: an up to date narrative review. Curr Opin Psychiatry 30:312-317

9. Leinonen A, Seikkula J, Alasuutari M (2016) Can i tell just by myself? Discussing a parental mental disorder with a child in a research interview. Int J Child Youth Family Stud 7:125-147

10. Lenz A (2014) Kinder psychisch kranker Eltern. Hogrefe, Göttingen, Bern, Wien

11. Lohaus A, Nussbeck FW (2016) FRKJ 8-16 Fragebogen zu Ressourcen im Kindes- und Jugendalter

12. Mayring P (2015) Qualitative Inhaltsanalyse: Grundlagen und Techniken. Beltz, Weinheim

13. Petermann F, Schmidt MH (2006) Ressourcen - ein Grundbegriff der Entwicklungspsychologie und Entwicklungspsychopathologie? Kindh Entwickl 15:118-127

14. Pretis M, Dimova A (2016) Frühförderung mit Kindern psychisch kranker Eltern. Ernst Reinhardt Verlag, München, Basel

15. Reinisch A, Schmuhl M (2012) Das Präventionsprojekt für Kinder psychisch erkrankter Eltern „Kanu - Gemeinsam weiterkommen". In: Bauer U (Hrsg) Prävention für Familien mit psychisch erkrankten Eltern: Bedarf, Koordination, Praxiserfahrung. SpringerVS, Wiesbaden, S119-138

16. Schmenger S, Schmutz E (2018) Überblick über Angebote, Initiativen und Unterstützungsmaßnahmen zur Verbesserung der Situation von Kindern und Jugendlichen aus Familien mit einem psychisch erkrankten oder suchterkrankten Elternteil

17. Staets S, Gumz H (2008) Kipkel - Kinder psychisch kranker Eltern. Psychoprax Neuroprax 11:26-29

18. Thanhäuser $M$, Lemmer $G$, de Girolamo $G$ Christiansen H (2017) Do preventive interventions for children of mentally ill parents work? Results of a systematic review and meta-analysis. Curr Opin Psychiatry 30:283-299

19. Thomas G (2016) How to do your case study. SAGE, Los Angeles, London

20. Trautmann T (2010) Interviews mit Kindern:Grundlagen, Techniken, Besonderheiten, Beispiele. VS, Wiesbaden

21. Trondsen MV (2012) Living with a mentally ill parent: exploring adolescents' experiences and perspectives. Qual Health Res 22:174-188

22. WilleN, BettgeS, Ravens-SiebererU (2008) Riskand protective factors for children's and adolescents' mental health: results of the BELLAstudy. Eur Child Adolesc Psychiatry 17:133-147 